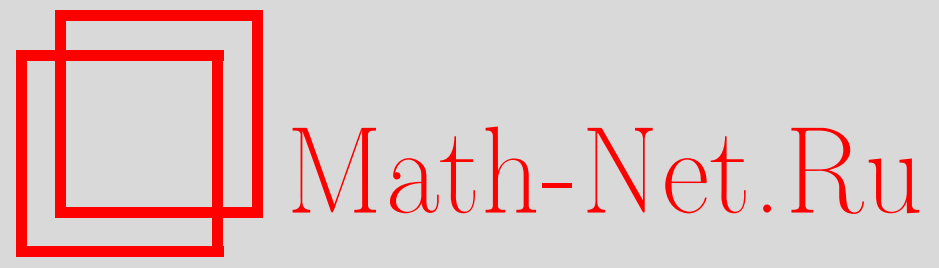

А. Г. Баскаков, К. И. Чернышов, Линейные отношения, дифференциальные включения и вырожденные полугруппы, Функи. анализ и его прил., 2002, том 36, выпуск 4, 65-70

DOI: https://doi.org/10.4213/faa220

Использование Общероссийского математического портала MathNet.Ru подразумевает, что вы прочитали и согласны с пользовательским соглашением

http: //www . mathnet.ru/rus/agreement

Параметры загрузки:

IP : 54.224 .187 .69

26 апреля 2023 г., 13:37:02

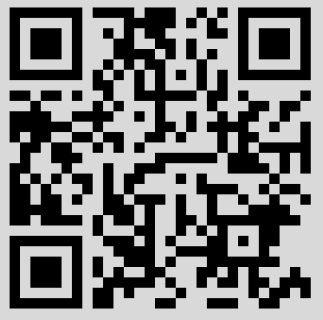




\title{
Линейные отношения, дифференциальные включения и вырожденные полугруппы*
}

\author{
(C) 2002. А. Г. БАСКАКОВ, К. И. ЧЕРНЫШОВ
}

Пусть $X$ - комплексное банахово пространство. Линейным отношением на $X$ называется линейное подпространство $A \subset X \times X$. Введем в рассмотрение основные понятия из теории линейных отношений и в связи с этим упомянем о двух недавно вышедших монографиях $[1,2]$, где имеется обширная библиография. В определении линейного отношения мы следуем А. Аренсу [3] и не всегда придерживаемся терминологии из $[1,2]$ (в частности, избегаем использовать термин «многозначный оператор»). Отметим, что приложения теории бинарных отношений в гильбертовом пространстве к теории дифференциальных операторов рассмотрены в [4].

Подпространство $D(A)=\{x \in X \mid$ существует $y \in X$, такой, что $(x, y) \in A\}$ из $X$ называется областью определения отношения $A \subset X \times X$. Через $A x$, где $x \in D(A)$, обозначается множество $\{y \in X \mid(x, y) \in A\}$, Ker $A=\{x \in D(A) \mid$ $0 \in A x\}-$ ядро отношения $A$ и $\operatorname{Im} A=\{y \in X \mid$ существует $x \in D(A)$, такой, что $(x, y) \in A\}-$ область его значений. Если линейное отношение $A \subset X \times X$ является графиком некоторого линейного оператора $\widetilde{A}: D(\widetilde{A}) \subset X \rightarrow X$, то в дальнейшем они отождествляются, и для их обозначения используется один и тот же символ $A$. Если $A \subset X \times X-$ линейное отношение, то отношение $A^{-1} \subset X \times X$ определяется равенством $A^{-1}=\{(y, x) \in X \times X \mid(x, y) \in A\}$.

Резольвентным множеством линейного отношения $A \subset X \times X$ называется множество $\rho(A)$ всех $\lambda \in \mathbb{C}$, для которых отношение $(A-\lambda I)^{-1} \subset X \times X$ принадлежит банаховой алгебре End $X$ линейных ограниченных операторов, действующих в $X$. Множество $\sigma(A)=\mathbb{C} \backslash \rho(A)$ называется спектром отношения $A$. Обычным образом доказывается, что множество $\rho(A)$ открыто, спектр $\sigma(A)$ замкнут, а его резольвента $R(\cdot)=R(\cdot, A): \rho(A) \rightarrow$ End $X, R(\lambda, A)=(A-\lambda I)^{-1}, \lambda \in \rho(A)$, является псевдорезольвентой в общепринятом смысле, причем $A 0=\operatorname{Ker} R\left(\lambda_{0}, A\right)$ для любого $\lambda_{0} \in \rho(A)$.

Если $B \in$ End $X-$ квазинильпотентный оператор, то линейное отношение $B^{-1} \subset X \times X$ имеет пустой спектр. Во избежание проблем, связанных с возможной пустотой спектра линейного отношения, далее используется следующее понятие.

Расширенным спектром (линейного) отношения $A \subset X \times X$ называется подмножество $\widetilde{\sigma}(A)$ из расширенной комплексной плоскости $\widetilde{\mathbb{C}}=\mathbb{C} \cup\{\infty\}$, которое совпадает с $\sigma(A)$, если $A 0=\{0\}$ (т.е. $A-$ линейный оператор), резольвента $R(\cdot, A)$ отношения $A$ допускает голоморфное расширение в точку $\infty$ и $R(\infty, A)=0$. В противном случае полагается $\widetilde{\sigma}(A)=\sigma(A) \cup\{\infty\}$. Множество $\widetilde{\rho}(A)=\widetilde{\mathbb{C}} \backslash \widetilde{\sigma}(A)$ называется расширенным резольвентным множеством отношения $A$. Отметим, что если $X \neq\{0\}$, то $\widetilde{\sigma}(A) \neq \varnothing$ для любого отношения $A \subset X \times X$, причем $\infty \in \widetilde{\sigma}(A)$, если $\operatorname{dim} A 0 \geqslant 1$.

\footnotetext{
*Работа выполнена при финансовой поддержке РФФИ, проект 01-01-00408.
} 
Линейные отношения могут возникать при исследовании линейных дифференциальных уравнений с необратимым оператором при производной, разрешимость которых естественным образом сводится к дифференциальному включению с линейным отношением $A \subset X \times X$. Например, построение решений в [2] велось с использованием полугруппы линейных операторов, генератором которой служило отношение $A$. Однако ограничения на $A$ из [2] не позволяли рассматривать важные классы дифференциальных включений даже в конечномерном пространстве $X$ (см. замечание 2).

Teоpema 1. Пусть $A \subset X \times X-$ линейное отношение. Тогда

$$
\widetilde{\sigma}\left(A^{-1}\right)=\left\{\lambda^{-1} \mid \lambda \in \widetilde{\sigma}(A)\right\} .
$$

Следствие 1. Если $A \subset X \times X-$ линейное отношение $u \mu \in \rho(A)$, то $\sigma(R(\mu, A))=\left\{(\mu-\lambda)^{-1}, \lambda \in \widetilde{\sigma}(A)\right\}$.

Утверждение следствия 1 было получено в [1, Theorem V.4.2].

Следствие 2. Пусть $A \subset X \times X-$ линейное отношение. Тогда $\widetilde{\sigma}(A)=\{\infty\}$

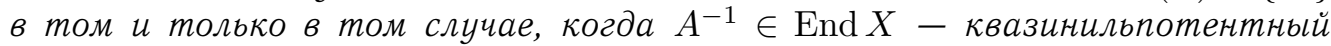
оператор (при этом $A^{-1}$ - нильпотентный оператор, если $R(\cdot, A)-$ многочлен).

СледствиЕ 3. Для линейного отношения $A \subset X \times X$ следующие условия эквивалентны:

1) $A \in$ End $X$;

2) $\infty \notin \widetilde{\sigma}(A)$;

3) $0 \notin \sigma\left(A^{-1}\right)$.

ЗАмЕчАНИЕ 1. Любая псевдорезольвента $\mathscr{R}: U \subset \mathbb{C} \rightarrow \operatorname{End} X$, определенная на открытом множестве $U$ из $\mathbb{C}$, является резольвентой линейного отношения $A=\left(\mathscr{R}\left(\lambda_{0}\right)\right)^{-1}+\lambda_{0} I$, где $\lambda_{0} \in U$, причем $\rho(A) \supset U$ и отношение $A$ не зависит от выбора числа $\lambda_{0}$. Кроме того, для любого числа $\mu_{0} \in \mathbb{C}$ любой оператор $B \in$ End $X$ может быть представлен в виде $B=R\left(\mu_{0}, B^{-1}+\mu_{0} I\right)$.

Доказательство теоремы 3.6 в [5] может быть основано на утверждении из замечания 1 .

Далее считается выполненным

ПРЕДПОЛОЖЕНИЕ 1. Резольвентное множество $\rho(A)$ линейного отношения $A \subset$ $X \times X$ непусто.

Теорема 2. Для линейного отношения $A \subset X \times X$ следующие условия эквивалентны:

1) точка $\infty$ является полюсом порядка $m-2$ функции $R(\cdot, A)$;

2) $A^{m-1} 0 \subset A^{m} 0=A^{m+1} 0, D\left(A^{m-1}\right) \supset D\left(A^{m}\right)=D\left(A^{m+1}\right)$ для некоторого $\lambda_{0} \in \rho(A)$ (включения строгие);

3) пространство $X$ представимо в виде прямой суммы $X_{0} \oplus X_{\infty}$ инвариантных относительно $A$ замкнутых подпространств $X_{0}, X_{\infty}$, причем сужение $A_{0}$ отношения $A$ на $X_{0}$ принадлежит End $X_{0}, \sigma\left(A_{0}\right)=\sigma(A)$ и, кроме того, $\widetilde{\sigma}\left(A_{\infty}\right)=\{\infty\}, A_{\infty}^{-1} \in$ End $X_{\infty},\left(A_{\infty}^{-1}\right)^{m}=0$ для сужения $A_{\infty}$ отношения $A$ на $X_{\infty}$, причем $\left(A_{\infty}^{-1}\right)^{m-1} \neq 0$.

Отметим, что если выполнено условие 2), то фигурирующие в исследовании подпространства имеют вид $X_{\infty}=A^{m} 0, X_{0}=D\left(A^{m}\right)$, причем $D\left(A^{m}\right)=$ $\operatorname{Im} R\left(\lambda_{0}, A\right)^{m}$ для любого $\lambda_{0} \in \rho(A)$. 
Рассмотрим дифференциальное операторное включение

$$
\begin{gathered}
\dot{x}(t) \in A x(t), \quad t \in \mathbb{R}_{+}=[0 ;+\infty), \\
x(0)=x_{0} \in D(A),
\end{gathered}
$$

где $A \subset X \times X-$ линейное отношение. Дифференцируемая функция $x: \mathbb{R}_{+} \rightarrow X$, для которой $x(0)=x_{0}, x(t) \in D(A)$ для любого $t>0$, называется решением задачи Коши (1)-(2), если она удовлетворяет включению (1).

Замыкание множества начальных условий вида (2), для которых существует решение задачи (1)-(2), назовем фазовым пространством дифференциального включения (1) и обозначим его символом $\Phi_{A}$.

Теорема 3. Если отношение $A \subset X \times X$ удовлетворяет одному из условий теоремь 2 , то каждое решение $x: \mathbb{R}_{+} \rightarrow X$ задачи (1)-(2) с $x_{0} \in X_{0}$ определяется с помощью аналитической группы операторов $\left\{\exp A_{0} t, t \in \mathbb{R}\right\}$ u имеет вид $x(t)=\left(\exp A_{0} t\right) x_{0}, t \in \mathbb{R}$, m.e. $\Phi_{A}=X_{0}$.

Далее построение фазового пространства $\Phi_{A}$ будем осуществлять в условиях следующего предположения относительно отношения $A$.

ПредПоложениЕ 2. Существует число $m \in \mathbb{N}$ и последовательность $\left\{\lambda_{n}\right\} \subset$ $\rho(A)$, такие, что

1) $\lim _{n \rightarrow \infty}\left|\lambda_{n}\right|=\infty$

2) $\sup _{n \geqslant 1}\left\{\left|\lambda_{n}^{m}\right| \cdot\left\|\left(R\left(\lambda_{n}, A\right)\right)^{m}\right\|\right\}<\infty$.

Предположение 2 , например, выполнено, если имеет место одно из условий теоремы 2.

В условиях предположения 2 введем в рассмотрение ограниченную последовательность операторов

$$
\mathscr{A}_{n}=I-\left(-\lambda_{n} R\left(\lambda_{n}, A\right)\right)^{m}, \quad n \geqslant 1,
$$

из алгебры End $X$ и (замкнутое) подпространство

$$
\widetilde{X}=\left\{x \in X: \text { существует } \lim _{n \rightarrow \infty} \mathscr{A}_{n} x\right\} .
$$

Это подпространство инвариантно относительно $A$, и поэтому можно рассмотреть сужение $\widetilde{A}$ отношения $A$ на $\widetilde{X}$.

ЛЕмма 1. Подпространство $\widetilde{X}$ в условиях предположения 2 допускает прямое разложение вида

$$
\widetilde{X}=X_{m} \oplus X^{(m)},
$$

где $X_{m}, X^{(m)}$ - замкнутые инвариантные относительно $A$ подпространства, $X_{m}=A^{m} 0, u$

$$
\widetilde{A}=A_{m} \oplus A^{(m)},
$$

где $A_{m}, A^{(m)}-$ сужения отношения $\widetilde{A}$ на $X_{m}, X^{(m)}$ соответственно, $\widetilde{\sigma}\left(A_{m}\right)=$ $\{\infty\}, A_{m}^{-m}=0$ и $A^{(m)}$ - линейный замкнутый оператор со спектром $\sigma\left(A^{(m)}\right)=$ $\sigma(\widetilde{A})$.

Обозначим через $X^{*}$ сопряженное к $X$ банахово пространство и через $A^{*} \subset$ $X^{*} \times X^{*}$ сопряженное к $A$ линейное отношение (см. [2, Sec. 1.5]).

Теорема 4. Пусть выполнено предположение 2. Для того итобы выполнялось равенство $\widetilde{A}=A$ (т.е. $\widetilde{X}=X)$, необходимо и достаточно, итобы векто- 
ры из подпространства $X_{m}=A^{m} 0$ разделяли функциональ из подпространства $X_{m}^{*}=\left(A^{*}\right)^{m} 0$. В частности, $\widetilde{X}=X$, если выполнено одно из следующих условий:

1) $X$ - рефлексивное банахово пространство;

2) $R\left(\lambda_{0}, A\right) \in$ End $X$ - слабо компактный оператор при некотором $\lambda_{0} \in$ $\rho(A)$

3) $\operatorname{dim} X_{m}=\operatorname{dim} X_{m}^{*}<\infty$.

ПредПоложЕниЕ 3. Существуют такие числа $M>0, \omega \in \mathbb{R}, m \in \mathbb{N}$, что для всех $\lambda \in \mathbb{C}$ с $\operatorname{Re} \lambda>\omega$ и всех $n \in \mathbb{N}$ имеют место оценки (типа ХиллеФиллипса-Иосиды)

$$
\left\|(R(\lambda, A))^{m n}\right\| \leqslant \frac{M}{(\operatorname{Re} \lambda-\omega)^{m n}}, \quad n \in \mathbb{N} .
$$

Если выполнено предположение 3, то предположение 2 справедливо для любой последовательности $\left(\lambda_{n}\right)$ из $\mathbb{R}_{+}$со свойством $\lim _{n \rightarrow \infty} \lambda_{n}=\infty$.

Сильно непрерывную полугруппу линейных операторов $\{T(t) ; t \geqslant 0\}$ из алгебры End $X$ назовем вырожденной полугруппой операторов класса $C_{0}\left(C_{0}\right.$-полугруппой $)$, если $T(0) \neq I$. В этом случае определение полугруппы влечет за собой равенство $T(0)=P_{1}$, где $P_{1}-$ проектор на подпространство $X_{1}=\operatorname{Im} P_{1}$. Сужение полугруппы $T$ на $X_{1}$ определяет $C_{0}$-полугруппу операторов $\left\{T_{1}(t) ; t \geqslant 0\right\}$ из End $X_{1}$ и поэтому существуют постоянные $M>0, \omega \in \mathbb{R}$, такие, что $\|T(t)\| \leqslant M \exp \omega t, t \geqslant 0$. Функция

$$
R(\lambda)=\int_{0}^{\infty} e^{-\lambda t} T(t) d t, \quad \operatorname{Re} \lambda>\omega,
$$

является псевдорезольвентой, и, следовательно, существует единственное линейное отношение $A \subset X \times X$, резольвентой которого является функция $R$. Это линейное отношение назовем генератором вырожденной полугруппы $\{T(t) ; t \geqslant 0\}$. Нетрудно видеть, что $\operatorname{Ker} T(0)=A 0$.

Обратимся к включению (1). Пусть выполнено предположение 2 (или, в частности, предположение 3 ), причем $\operatorname{dim} A 0 \geqslant 1$, т. е. $A \subset X \times X-$ линейное отношение, не являющееся линейным оператором. Вырожденную полугруппу операторов $\{\widetilde{T}(t) ; t \geqslant 0\}$ из алгебры End $\widetilde{X}$ назовем разрешающей вырожденной полугруппой для включения $(1)$ на подпространстве $X^{(m)}$ из $\widetilde{X}$, если выполнены следующие два условия:

1) $x(t)=\widetilde{T}(t) x_{0}, t \in \mathbb{R}_{+},-$решение включения (1) при любом векторе $x_{0}$ из некоторого плотного в $X^{(m)}$ подпространства;

2) $\widetilde{T}(0)=P^{(m)}$, где $P^{(m)} \in$ End $\widetilde{X}-$ проектор, отвечающий разложению $(3)$ подпространства $\widetilde{X}$ (т. е. $\operatorname{Im} P^{(m)}=X^{(m)}$ и $\operatorname{Ker} P^{(m)}=X_{m}$ ).

Непосредственно из этого определения следует, что генератором сужения полугруппы $\{\widetilde{T}(t) ; t \geqslant 0\}$ на $X^{(m)}$ служит сужение $A^{(m)}$ отношения $A$ на $X^{(m)}$, которое, согласно лемме 1 , является линейным замкнутым оператором. Таким образом, справедлива

Теорема 5. Пусть выполнено предположение $3 u \operatorname{dim} A 0 \geqslant 1$. Тогда $\Phi_{A} \cap$ $\widetilde{X}=X^{(m)}$ и существует единственная разрешающая полугруппа операторов $\{\widetilde{T}(t) ; t \geqslant 0\} \subset$ End $\widetilde{X}$ для включения (1) на подпространстве $\widetilde{X}$, генератором 
которой служит сужение $A^{(m)}$ отношения $A$ на $X^{(m)}$. Если векторы из подпространства $X_{m}=A^{m} 0 \subset X$ разделяют функциональ из подпространства $X_{m}^{*}=\left(A^{*}\right)^{m} 0 \subset X^{*}, \operatorname{mo}\{\widetilde{T}(t) ; t \geqslant 0\}-$ вырожденная разрешающая полугруппа операторов на банаховом пространстве $X=\widetilde{X}$. В частности, если $A^{m} 0$ - конечномерное подпространство из $X$, mо $_{A}=X^{(m)}$.

Теорема 6. Пусть выполнено предположение 2 и существуют числа $M>0$, $\omega \in \mathbb{R}$, такие, ито для всех $x \in X^{(m)}, \lambda \in \mathbb{C} c \operatorname{Re} \lambda>\omega$ исех $n \in \mathbb{N}$ имеют место оценки

$$
\left\|(R(\lambda, A))^{n} x\right\| \leqslant \frac{M\|x\|}{(\operatorname{Re} \lambda-\omega)^{n}} .
$$

Тогда справедливы все утверждения теоремы 5.

Линейное отношение $A \subset X \times X$ называется секториальным или $m$-секториальным (ср. [6]), если для некоторых $a \in \mathbb{R}, \theta \in(\pi / 2, \pi), M \geqslant 1$ сектор $\Omega=\Omega_{a, \theta}=\{\lambda \in \mathbb{C}:|\arg (\lambda-a)|<\theta, \lambda \neq a\}$ содержится в резольвентном множестве $\rho(A)$ отношения $A$ и существует такое $m \in \mathbb{N}$, что

$$
\left\|(R(\lambda, A))^{m}\right\| \leqslant M|\lambda-a|^{-m} \text { для любого } \lambda \in \Omega_{a, \theta} \text {. }
$$

Отметим, что для гильбертовых пространств соответствующее определение дано в статье [7]; там же были получены некоторые свойства секториальных отношений. Данное нами определение секториального отношения отличается знаком от общепринятого определения секториального оператора в гильбертовом пространстве [8].

ПредПоложениЕ 4. Отношение $A \subset X \times X$ является секториальным.

Выберем жорданов контур $\gamma$, окружающий $\sigma(A)$, так, чтобы он при достаточно больших значениях $|\lambda|$ лежал на лучах $|\arg \lambda|=\theta_{1}=\theta-\varepsilon \in(\pi / 2, \pi)$ при некотором $\varepsilon>0$.

Введем в рассмотрение полугруппу операторов $\{T(t) ; t>0\}$ с помощью формулы

$$
T(t)=-\frac{1}{2 \pi i} \int_{\gamma} e^{\lambda t} R(\lambda, A) d \lambda .
$$

Корректность определения в (5) вытекает из оценки $\|R(\lambda, A)\| \leqslant M_{1}|\lambda-a|^{m-2}$ для любого $\lambda \in \Omega_{a, \theta}$ с $M_{1}>0$, которая следует из (4) после $m$-кратного интегрирования правой части формулы (5) и замены $\lambda=\mu t$.

Отметим, что для секториального отношения $A \subset X \times X$ выполнено предположение 2 , и поэтому можно рассматривать замкнутое подпространство $\widetilde{X}$ и разложение $\widetilde{X}=X_{m} \oplus X^{(m)}$. Также верна теорема 4 об условиях, обеспечивающих выполнение равенства $\tilde{X}=X$.

ТЕОремА 7. В условиях предположения 4 справедливы следующие утверждения:

1) $X^{(m)}=\left\{x \in X \mid \lim _{t \rightarrow+0} T(t) x=x\right\}, X_{m}=\bigcap_{t>0} \operatorname{Ker} T(t)$;

2) сужение $\left\{T^{(m)}(t) ; t \geqslant 0\right\}$ nолугруппь $\{T(t) ; t \geqslant 0\}$ на подпространство $X^{(m)}$ является сильно непрерьвной при $t \geqslant 0$ полугруппой, $T(0)=I^{(m)}$ тождественный оператор в $X^{(m)}$, причем $\left\{T^{(m)}(t)\right\}$ допускает голоморфное расширение в некоторый сектор, содержащий $\mathbb{R}_{+}$. 
А. Г. Баскаков, К. И. Чернышов

ЗАмЕчАниЕ 2. Задача Коши $x(0)=x_{0} \in X$ для линейного дифференциального уравнения

$$
F \dot{x}=G x, \quad t \in \mathbb{R}_{+},
$$

с парой линейных замкнутых (в частности, ограниченных) операторов, действующих из банахова пространства $X$ в банахово пространство $Y$, при $\operatorname{Ker} F \neq\{0\}$ стандартным образом сводится к дифференциальному включению вида (1), где $A=F^{-1} G \subset X \times X$, и такой прием систематически используется в монографии [2] (см. также [9]).

В [2] в отличие от сделанных нами предположений 2-4 считались выполненными аналоги этих предположений при $m=1$ (см., например, $\$ 3$ гл. 1 и 1 гл. 3). Отметим, что условие $m=1$ не позволяет рассматривать дифференциальное уравнение вида (6) даже в конечномерном пространстве $X$, если, например, предположение 2 выполнено с $m=\min \left\{k \in \mathbb{N} \mid \operatorname{dim} A^{k} 0=\operatorname{dim} A^{k+1} 0\right\}>1$. Кроме того, в [2] отсутствуют условия (кроме условия рефлексивности исходного пространства), обеспечивающие выполнение равенства $\tilde{X}=X$. Отметим, что это равенство для гильбертова пространства получено в статье [7].

ЗАмЕчАнИЕ 3. Случай $m>1$ изучался в [9] методами $n$-интегрированных полугрупп, причем основные результаты приведены при априорном требовании $X=X_{m} \oplus X^{(m)}$ (в обозначениях из леммы 1 ).

В заключение отметим, что результаты настоящей заметки базируются на проведенных авторами исследованиях [10-14] по эргодической теории и разрешимости дифференциальных уравнений вида (6).

\section{ЛиТЕРАТУРА}

1. Cross R. Multivalued linear operators. M. Dekker, New York, 1998. 2. Favini A., Yagi A. Degenerate differential equations in Banach spaces. Pure and Applied Mathematics: A Series of Monographs and Textbooks, Vol. 215. M. Dekker. New York, 1998. 3. Arens R. Pacific J. Math., 11, 9-23 (1961). 4. Ахиезер Н. И., Глазман И. М. Теория линейных операторов в гильбертовом пространстве. Т. 2. Изд-во Харьк. гос. ун-та, Харьков, 1978. 5. Arendt $W$. Tübinger Berichte fur Funktionalanalysis, 9, 33-46 (1999/2000). 6. Хeнри Д. Геометрическая теория полулинейных параболических уравнений. Мир, М., 1985. 7. Рофе-Бекетов Ф. С. ДАН СССР, 255, №5, 1054-1058 (1980). 8. Като T. Теория возмущений линейных операторов. Мир, М., 1972. 9. Мельникова И. В., Гладченко А. В. Докл. РАН, 361, №6, 736-739 (1998). 10. Баскаков А. Г. Изв. вузов, сер. матем., № 11 (318), 3-11 (1988). 11. Баскаков А. Г., Чернышов К. И. Изв. РАЕН. МММИУ, 2, № 3, 39-69 (1998). 12. Баскаков А. Г., Чернышиов К. И. Изв. РАЕН. МММИУ, 3, № 3, 524 (1999). 13. Баскаков А. Г., Черныциов К. И. Докл. РАН, 371, № 3, 295-298 (2000). 14. Baskakov A. G., Chernyshov K. I. In: Spectral and Evolution Problems, Vol. 11 (Proc. of the Eleventh Crimean Autumn Math. School), Natl. Taurida Univ. "V. Vernadsky", Simferopol, 2001, pp. 136-143.

Воронежский государственный университет e-mail: pmmmmio@main.vsu.ru

Поступило в редакцию 1 ноября 2001 г.

Воронежская государственная лесотехническая академия

e-mail: chern@vsau.ru 